\title{
Improvement of thoracic aortic vasoreactivity by continuous and intermittent exercise in high-fat diet-induced obese rats
}

\author{
HONGPENG LIU ${ }^{1,2^{*}}$, ZHEN YANG $^{1 *}$, JIAN HU $^{1}$, YAN LUO $^{1}$, \\ LINGQIN ZHU $^{3}$, HUIFANG YANG ${ }^{3}$ and GUANGHUA LI ${ }^{1}$ \\ ${ }^{1}$ Department of Physiology, School of Basic Medical Science; ${ }^{2}$ Institute of Laboratory Medicine; \\ ${ }^{3}$ Public Health School, Ningxia Medical University, Yinchuan, Ningxia 750004, P.R. China
}

Received February 25, 2015; Accepted April 6, 2015

DOI: $10.3892 /$ br.2015.451

\begin{abstract}
The aim of the present study was to explore the effects of continuous and intermittent exercise on the thoracic aortic vasoreactivity and free radical metabolism in rats fed with a high-fat diet (HD). Sprague-Dawley (SD) rats were randomly divided into four groups ( $n=8$, each group): Conventional diet (CD), HD, HD with continuous exercise (HCE) and HD with intermittent exercise (HIE). HCE rats swam once/day for $90 \mathrm{~min}$; HIE rats performed swimming exercises 3 times/day, 30 min each time with an interval of $4 \mathrm{~h}$. In these two groups, the exercise was conducted 5 days a week for 8 weeks. Rats in the CD and HD groups were fed without swimming training. At the end of the exercise, all the rats were sacrificed and the blood, thoracic aorta and myocardium were collected immediately. The thoracic aortic vasoreactivity, the plasma total cholesterol (TC), triglyceride (TG), high-density lipoprotein (HDL), low-density lipoprotein (LDL), superoxide dismutase (SOD), malondialdehyde (MDA) and vascular endothelial nitric oxide synthase (eNOS) gene expression were measured. Compared to the control group, in the HD group the enhanced contractile response of the thoracic aortic rings to noradrenaline (NA) was observed $(\mathrm{P}<0.01)$. The levels of TC and LDL $(\mathrm{P}<0.01)$ were also increased in serum while the HDL level was reduced without statistical significance. In addition, the MDA content was upregulated in the myocardium, but the SOD level decreased $(\mathrm{P}<0.01)$. Furthermore, the expression of vascular eNOS mRNA was reduced $(\mathrm{P}<0.01)$. However, following the exercise the contraction of the thoracic aorta vascular rings to NA was reduced in the HCE and HIE
\end{abstract}

Correspondence to: Professor Guanghua Li, Department of Physiology, School of Basic Medical Science, Ningxia Medical University, 1160 Shengli Street, Yinchuan, Ningxia 750004, P.R. China

E-mail: ligh@nxmu.edu.cn

${ }^{*}$ Contributed equally

Key words: continuous and intermittent exercise, high-fat diet, vascular reactivity groups $(\mathrm{P}<0.01)$, and the decreased contractile response was more evident in the HIE group compared to the HCE group $(\mathrm{P}<0.01)$. Additionally, in the HCE group the level of TG $(\mathrm{P}<0.01)$ was decreased, while the HDL $(\mathrm{P}<0.01)$ level was increased. Although the reduction of the TC and LDL level was also observed there was no significant difference compared to the HD group. In the HIE group, the TG, TC and LDL were downregulated while the HDL was enhanced $(\mathrm{P}<0.01)$. The TC and LDL levels were decreased more than those of the HCE group; however, there was no significant difference in the TG and HDL levels between these two groups; additionally, in these two exercise groups, the MDA level was decreased in the myocardium $(\mathrm{P}<0.01)$ while the SOD level was increased $(\mathrm{P}<0.01)$. Furthermore, the expression of eNOS was upregulated $(\mathrm{P}<0.01)$, but the increase was much more in the HIE group than that in the HCE group. In conclusion, exercise may attenuate the aggravated contraction induced by NA and improve the activity of the thoracic aorta in obese rats, which may be associated with enhanced antioxidant enzyme activity and reduced free radical generating. Additionally, intermittent exercise is better than the continuous exercise in improving the thoracic aorta vasoreactivity.

\section{Introduction}

Obesity is a serious public health problem worldwide (1), which is often accompanied by excessive visceral fat, dyslipidemia, hypertension, diabetes, coronary arteriosclerotic heart disease, cancer, non-alcoholic fatty liver disease, cardiovascular disease and other chronic diseases $(2,3)$. Arterial elasticity is an important factor in predicting the cardiovascular risk. One of the most basic and direct indicators reflecting the functional status of artery blood vessels is vascular compliance (4). Studies have shown that a high-fat diet (HD) and lack of physical activity are the most important factors for the development of obesity. Long-term aerobic exercise markedly improved the abnormal hemorheological property and the oxidative stress in rats with hypercholesterolemia. It has been shown that aerobic exercise plays an important role in anti-free radicals, lipid peroxides, prevention and treatment of cardiovascular disease $(5,6)$. However, regular aerobic exercise for a long period and short exhaustive exercise have different effects on vasoreactivity (7). Exercise 
intensity, frequency, duration and different diet have various effects on the metabolic activity (8). Therefore, sports as a non-drug therapy is an important method to control obesity and other complications, and this research has attracted increasing attention. The present study was designed to evaluate the effect of the continuous and intermittent exercise on the contraction of thoracic aortic vascular rings and metabolism of free radicals in rats fed with HD and to explore the different mechanisms of exercise on the cardiovascular function status.

\section{Materials and methods}

Experimental animals. A total of 32 male Sprague-Dawley (SD) rats $(180 \pm 10 \mathrm{~g})$ were bred (provided by the Experimental Animal Center of Ningxia Medical University, Yinchuan, Ningxia, China) for 8 weeks with a free diet and water ad libitum, and a temperature of $32 \pm 1^{\circ} \mathrm{C}$ with 12 -h light. Rats were randomly divided into four groups: Conventional diet (CD), HD, HD with continuous exercise (HCE) and HD with intermittent exercise (HIE). There were 8/cage and rats with a poor ability for swimming were eliminated. The experimental procedures were approved by the Animal Ethics Committee of the Ningxia Medical University and Use Committee in accordance with the guidelines of the Council of the Physiological Society of China.

Preparation of the experimental animal models. In the control group, rats were fed with CD: $23 \mathrm{~g}$ protein, $49 \mathrm{~g}$ carbohydrate, $4 \mathrm{~g}$ fat, $5 \mathrm{~g}$ fiber, $7 \mathrm{~g}$ bone meal and $6 \mathrm{~g}$ vitamins/100 g. In the HD group, rats were fed with the high-fat along with the standard diet: Peanuts, milk chocolate and sweet biscuits in a ratio of 3:2:2:1. Protein accounted for $20 \%$, fat for $20 \%$, sugar for $48 \%$ and cellulose for $4 \%$. Calories in the fat diet were $5.12 \mathrm{kcal} / \mathrm{g}$ (equivalent to $35 \%$ fat calories), while there was $4.07 \mathrm{kcal} / \mathrm{g}$ in the standard diet.

Exercise training protocol. The rats in the CD and HD groups were fed at room temperature without swimming training. The rats in the exercise groups swam in a plastic pool with a diameter of $60 \mathrm{~cm}$, the water depth was $55 \mathrm{~cm}$ and temperature was $28-32^{\circ} \mathrm{C}$. For the continuous exercise, rats swam for 30 , 60 and $90 \mathrm{~min}$ in the first, second and third day, respectively, then the rats swam one time for $90 \mathrm{~min} /$ day. For the intermittent exercise, rats performed intermittent swimming for 10 , 20 and $30 \mathrm{~min}$ in the first, second and third day, respectively, and the total swimming time throughout the experiment was $90 \mathrm{~min} /$ day, which was divided into three time periods and maintained at a 4-h interval for the following time schedule: The rats swam at 7:00 a.m., 11:30 a.m. and 3:30 p.m., respectively, for $30 \mathrm{~min}$ each time. Rats with intermittent exercise were reared in the respective cages. All the rats swam with a load of $5 \%$ of their body weight strapped to their tail. The exercise was conducted for 5 days a week for 8 weeks with moderate intensity.

Sample preparation. After 8 weeks of training, rats were rested and fasted for $24 \mathrm{~h}$. Subsequently the rats were anesthetized with $20 \%$ urethane $(0.5 \mathrm{ml} / \mathrm{kg})$ and the blood was taken from the heart. The serum was separated and stored at $-80^{\circ} \mathrm{C}$ for further use. The heart was obtained and the residual blood was washed with saline. Tissue homogenate (10\%) was prepared and the supernatant was stored at $-80^{\circ} \mathrm{C}$.

Recording the tension of the aortic vascular rings. At the same time, the thoracic aorta was isolated immediately and the connective tissue surrounding the blood vessels was carefully removed. The aorta was cut into 3-4 mm sections for measuring the tension. The vascular rings were hung in the isolated organ tissue bath with $10 \mathrm{ml} \mathrm{K}-\mathrm{H}$ solution $(10 \mathrm{mM} \mathrm{NaCl}, 4.6 \mathrm{mM}$ $\mathrm{KCl}, 2.5 \mathrm{mM} \mathrm{CaCl}_{2}, 24.8 \mathrm{mM} \mathrm{NaHCO}, 1.2 \mathrm{mM} \mathrm{KH}_{2} \mathrm{PO}_{4}$, $1.2 \mathrm{mM} \mathrm{MgSO}_{4}$ and $5.6 \mathrm{mM}$ glucose $)$ at $37^{\circ} \mathrm{C}(\mathrm{pH} 7.4)$ and continuously perfused with $95 \% \mathrm{O}_{2}$ and $5 \% \mathrm{CO}_{2}$. The resting tension was adjusted to $1 \mathrm{~g}$, after equilibration for $1 \mathrm{~h}$ with flushing every $15 \mathrm{~min}$. The maximum contraction tension was induced with $60 \mathrm{mmol} / \mathrm{KCl}$, and subsequently the cumulative dose-response curve for noradrenaline (NA) $\left(10^{-10}-10^{-5} \mathrm{M}\right)$ was examined. The $\mathrm{KCl}$-induced maximum contraction was regarded as $100 \%$, and the values of the NA-induced contraction were expressed as a percentage of the maximal contraction induced by $\mathrm{KCl}\left(10^{-10}-10^{-5} \mathrm{M}\right)$.

Detection of superoxide dismutase (SOD) and malondialdehyde (MDA) levels in the myocardium. Oxidative stress indices were measured to study whether the continuous and intermittent exercises reduced HD-induced oxidative stress. SOD was measured by thiobarbituric acid in the myocardium; MDA was determined using the WST-1 method.

Detection of high-density lipoprotein (HDL), low-density lipoprotein (LDL), triglycerides (TG) and total cholesterol (TC) levels in plasma. The levels of serum Lipid (TC, HDL, LDL and TG; Daiichi Pure Chemicals Co., Ltd., Tokyo, Japan) were measured using a microplate reader and ultraviolet spectrophotometry.

Expression of endothelial nitric oxide synthase (eNOS). Total RNA was extracted from the thoracic aorta according to the manufacturer's instructions for the Axygen RNA extraction kit (Axygen Biosciences, Union City, CA, USA). The concentration of the total RNA was determined by spectrophotometry, and quality was assessed using the A260/A280 nm ratio within 1.8-2.0. cDNA was synthesized using a reverse transcription kit (Beijing TransGen Biotech Co., Ltd., Beijing, China). Quantitative polymerase chain reaction (qPCR) was performed using a QuantiTect SYBR-Green PCR kit (Beijing TransGen Biotech Co., Ltd.) as follows: 40 cycles of denaturation at $94^{\circ} \mathrm{C}$ for $30 \mathrm{sec}$, annealing at $60^{\circ} \mathrm{C}$ for $30 \mathrm{sec}$ and extension at $72^{\circ} \mathrm{C}$ for $30 \mathrm{sec}$. Primers designed for eNOS and $\beta$-actin are shown in Table I. The relative levels of the gene expression are shown by the $2^{-\Delta \Delta \mathrm{Ct}}$ method: $\Delta \mathrm{Ct}=\mathrm{Ct}_{\text {(target gene) }}$ - $\mathrm{Ct}_{\text {(action gene) }} ; \Delta \Delta \mathrm{Ct}=\Delta \mathrm{Ct}_{\text {(sample) }}-\Delta \mathrm{Ct}_{\text {(control) }}$.

Statistical analysis. Statistical analysis was performed using SPSS 18.0 software (IBM Corp., Armonk, NY, USA). All the data are expressed as mean \pm standard deviation. Two-way analysis of variance (ANOVA) was used to evaluate any differences among groups; one-way ANOVA was used to analyze the remaining data. $\mathrm{P}<0.05$ was considered to indicate a statistically significant difference. 
Table I. GenBank accession code, primer sequences and predicted size of the amplified product.

\begin{tabular}{llcr}
\hline Gene & \multicolumn{1}{c}{ Primer sequences } & GenBank & bp \\
\hline eNOS & Forward: 5'-CACACTGCTAGAGGTGCTGGAA-3' & NM_021838 & 109 \\
& Reverse: 5'-TGCTGAGCTGACAGAGTAGTAC-3' & & 285 \\
& Forward: 5'-TCATGAAGTGTGACGTTGACATCCGT-3' & & \\
& Reverse: 5'-CCTAGAAGCATTTGCGGTGCAGGATG-3' & & \\
\hline
\end{tabular}

bp, base pairs; eNOS, endothelial nitric oxide synthase.

Table II. Influences of HCE and HIE on body weight of HD-induced fat rats.

\begin{tabular}{lcccccccc}
\hline Group & Week 1 & Week 2 & Week 3 & Week 4 & Week 5 & Week 6 & Week 7 & Week 8 \\
\hline CD & $181.89 \pm 3.14$ & $204.25 \pm 4.92$ & $228.56 \pm 7.33$ & $248.11 \pm 5.21$ & $265.78 \pm 4.66$ & $283.89 \pm 3.76$ & $310.33 \pm 5.77$ & $336.22 \pm 6.78$ \\
HD & $184.33 \pm 4.27$ & $224.89 \pm 5.23^{\mathrm{c}}$ & $268.56 \pm 4.25^{\mathrm{c}}$ & $303.30 \pm 6.83^{\mathrm{c}}$ & $340.22 \pm 6.55^{\mathrm{c}}$ & $377.30 \pm 5.29^{\mathrm{c}}$ & $415.50 \pm 10.53^{\mathrm{c}}$ & $449.30 \pm 12.00^{\mathrm{c}}$ \\
HCE & $183.53 \pm 2.61$ & $222.89 \pm 3.98$ & $266.11 \pm 4.23$ & $299.54 \pm 6.53$ & $335.56 \pm 4.19$ & $370.44 \pm 3.21^{\mathrm{a}}$ & $399.78 \pm 4.00^{\mathrm{b}}$ & $425.78 \pm 7.10^{\mathrm{b}}$ \\
HIE & $184.00 \pm 4.50$ & $217.87 \pm 3.83$ & $259.12 \pm 4.61$ & $295.00 \pm 6.86$ & $333.50 \pm 7.33$ & $365.50 \pm 4.29^{\mathrm{a}}$ & $392.87 \pm 5.81^{\mathrm{b}}$ & $411.37 \pm 4.28^{\mathrm{b}, \mathrm{d}}$ \\
\hline
\end{tabular}

${ }^{\mathrm{a}} \mathrm{P}<0.05$ and ${ }^{\mathrm{b}} \mathrm{P}<0.01$ vs. $\mathrm{HD} ;{ }^{\mathrm{c}} \mathrm{P}<0.01$ vs. $\mathrm{CD} ;{ }^{\mathrm{d}} \mathrm{P}<0.05$ vs. HCE. Values are expressed as mean \pm standard deviation (n=8). $\mathrm{HD}$, high-fat diet; $\mathrm{CD}$, conventional diet; HCE, HD with continuous exercise; HIE, HD with intermittent exercise.

Drugs and reagents. NA was provided by Shanghai Hefeng Pharmaceutical Co., Ltd., (Shanghai, China) batch no. H12020621; the SOD and MDA assay kits were provided by Nanjing Jian Cheng Bioengineering Institute (Nanjing, China); and lipid (TC, TG, HDL and LDL) kits were purchased from the Daiichi Pure Chemicals Co., Ltd.

\section{Results}

Influences of continuous and intermittent exercise on the body weight of obese rats. As shown in Table II, the body weight of the rats in each group increased every week. In the late stage of the swimming exercise, the weight increased more in the HD group than that in the control group $(\mathrm{P}<0.01)$; continuous and intermittent exercise (HCE and HIE groups, respectively) decreased the gain in body weight compared to that of the HD group $(\mathrm{P}<0.01)$, and additionally, the intermittent exercise was more effective than the continuous exercise $(\mathrm{P}<0.05)$.

Effects of continuous and intermittent exercise on the contraction of thoracic aortic vascular ring in HD rats. As shown in Fig. 1, contractile reactivity of thoracic aortic rings to NA increased with its increasing concentration in each group. The contractive response of thoracic aorta from rats fed with HD was significantly increased compared to that of the control group $(\mathrm{P}<0.01)$. The increased contraction induced by NA was attenuated by the continuous and intermittent exercise $(\mathrm{P}<0.01)$. Compared to the HCE group, this effect induced by the intermittent exercise in the HIE group was more evident $(\mathrm{P}<0.01)$.

Effects of continuous and intermittent exercise on the metabolism of free radicals in myocardium from rats fed with $H D$.

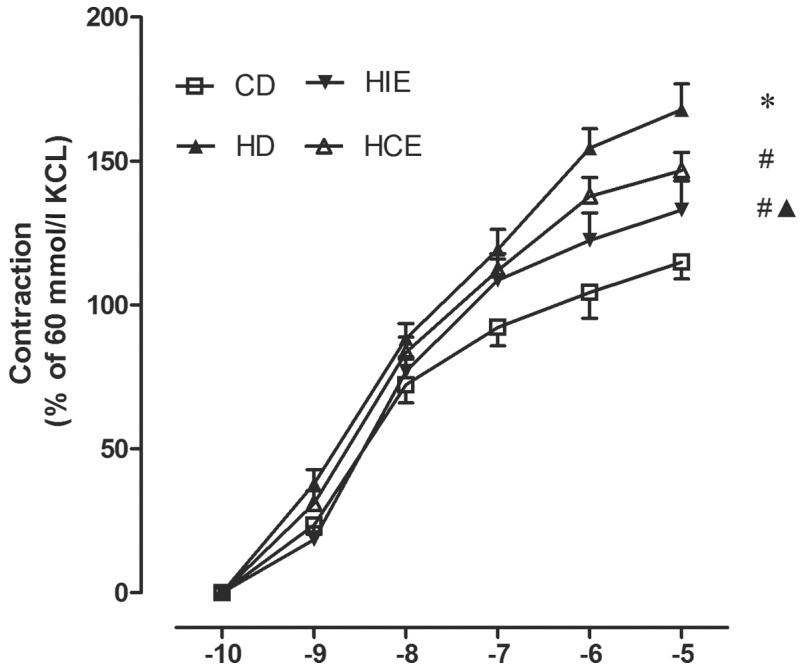

Figure 1. Contractile response of vascular ring to noradrenaline (NA). Dose-dependence of NA on the contraction of the thoracic aorta rings separated from rats in the conventional diet (CD), high-fat diet (HD), HD with continuous exercise (HCE) or HD with intermittent exercise (HIE) groups. The contraction induced by $60 \mathrm{mmol} / 1 \mathrm{KCl}$ was taken as $100 \%$. Data are expressed as mean \pm standard deviation $(\mathrm{n}=8) .{ }^{~} \mathrm{P}<0.01 \mathrm{vs}$. HD; ${ }^{*} \mathrm{P}<0.01$ vs. $\mathrm{CD} ;{ }^{\wedge} \mathrm{P}<0.01$ vs. HCE.

As shown in Fig. 2, the SOD activity of the myocardium significantly decreased in the HD group compared to that in the control group $(\mathrm{P}<0.01)$, and the continuous and intermittent exercise elevated the SOD activity significantly compared to that in the HD group $(\mathrm{P}<0.01)$. Furthermore, the myocardial SOD activity in the HIE group was higher than that in the HCE group, although there was no significant difference. The MDA level in the myocardium was also significantly increased in the HD group compared to that in the control group $(\mathrm{P}<0.01)$, but 
Table III. Effects of HCE and HIE on blood lipids in HD fed rats.

\begin{tabular}{lclcl}
\hline Group & \multicolumn{1}{c}{ TG } & \multicolumn{1}{c}{ TC } & \multicolumn{1}{c}{ HDL } & \multicolumn{1}{c}{ LDL } \\
\hline CD & $1.43 \pm 0.92$ & $1.61 \pm 0.24$ & $0.85 \pm 0.17$ & $0.33 \pm 0.10$ \\
HD & $1.58 \pm 0.87$ & $7.95 \pm 1.17^{\mathrm{b}}$ & $0.78 \pm 0.17$ & $6.45 \pm 1.64^{\mathrm{b}}$ \\
HCE & $0.75 \pm 0.30^{\mathrm{a}}$ & $6.90 \pm 1.85$ & $1.03 \pm 0.15^{\mathrm{a}}$ & $6.15 \pm 1.76$ \\
HIE & $0.40 \pm 0.18^{\mathrm{a}}$ & $2.75 \pm 1.09^{\mathrm{a}, \mathrm{c}}$ & $1.04 \pm 0.08^{\mathrm{a}}$ & $2.00 \pm 0.82^{\mathrm{a}, \mathrm{c}}$
\end{tabular}

${ }^{\mathrm{a}} \mathrm{P}<0.01$ vs. $\mathrm{HD} ;{ }^{\mathrm{b}} \mathrm{P}<0.01$ vs. $\mathrm{CD} ;{ }^{\mathrm{c}} \mathrm{P}<0.01$ vs. $\mathrm{HCE}$. Values are expressed as mean \pm standard deviation $(n=8)$. $H D$, high-fat diet; $C D$, conventional diet; HCE, HD with continuous exercise; HIE, HD with intermittent exercise; TG, triglyceride; TC, total cholesterol; HDL, high-density lipoprotein; LDL, low-density lipoprotein.

A

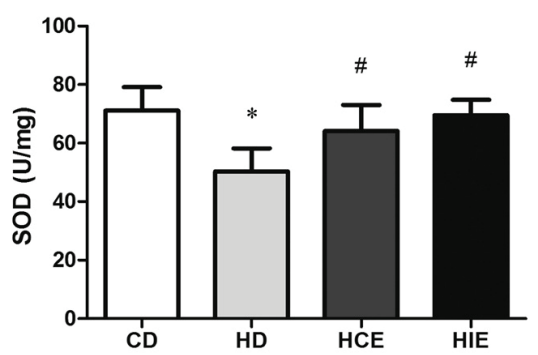

B

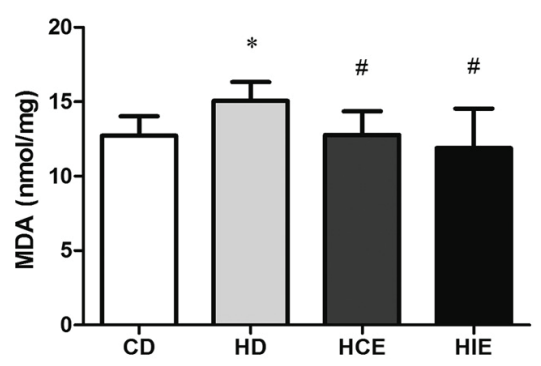

Figure 2. Effects of high-fat diet (HD) with continuous exercise (HCE) and HD with intermittent exercise (HIE) on (A) superoxide dismutase (SOD) and (B) malondialdehyde (MDA) metabolism in rats fed with HD. The values are expressed as mean \pm standard deviation $(n=8) .{ }^{~} \mathrm{P}<0.01$ vs. HD ${ }^{*} \mathrm{P}<0.01$ vs. conventional diet (CD).

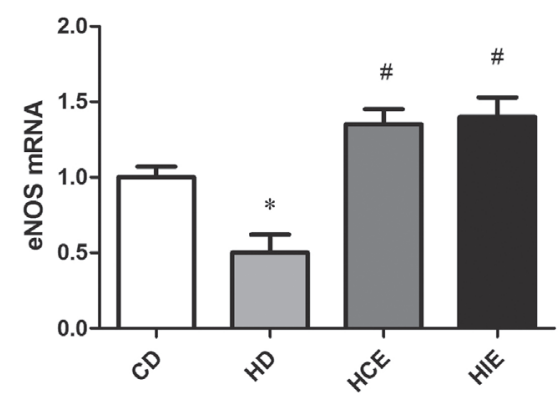

Figure 3. Effects of high-fat diet (HD) with continuous exercise (HCE) and HD with intermittent exercise (HIE) on the expression of endothelial nitric oxide synthase (eNOS) mRNA in the rats fed with HD. Data are expressed as mean \pm standard deviation $(\mathrm{n}=8) .{ }^{~} \mathrm{P}<0.01$ vs. $\mathrm{HD} ;{ }^{*} \mathrm{P}<0.01$ vs. conventional $\operatorname{diet}(\mathrm{CD})$.

following the exercise, the MDA content was reduced $(\mathrm{P}<0.01)$. The MDA level was also lower in the HIE group compared to the HCE group.
Effects of continuous and intermittent exercise on plasma lipid metabolism in rats fed with HD. As shown in Table III, the lipid metabolism level could be affected by continuous and intermittent exercise. TC and LDL levels significantly increased in the HD group $(\mathrm{P}<0.01)$. Compared to the HD group, the level of TG $(\mathrm{P}<0.01)$ decreased in the HCE group, while the HDL $(\mathrm{P}<0.01)$ level increased, the reduction of the TC and LDL levels were also observed but there was no statistical significance. In the HIE group, the TG, TC and LDL decreased and the HDL increased $(\mathrm{P}<0.01)$. Of note, the TC and LDL levels decreased more in the HIE group than that in the HCE group.

Expression of eNOS mRNA. As shown in Fig. 3, the expression level of vascular eNOS mRNA in the HD group $(\mathrm{P}<0.01)$ was reduced compared to that in the normal diet group. However, its expression level in the HCE and HIE groups was upregulated compared to that of the HD group $(\mathrm{P}<0.01)$, but there was no statistical significance between the HCE and HIE groups $(\mathrm{P}>0.05)$.

\section{Discussion}

In the present study, the contractive response of the thoracic aortic rings to NA was significantly increased in the HD group, but following exercise, this increased contraction induced by NA was attenuated. In addition, the effect of intermittent exercise on thoracic aortic vasoreactivity was more evident than that of the continuous exercise. These findings were consistent with a previous study (9). With the improvement of living conditions, HD has become one of the important factors that lead to obesity. Obesity is well-known as a risk factor for cardiovascular events. Studies have demonstrated that damaged aortic endothelium and abnormal lipid metabolism appear in rats fed with HD (10). Furthermore, HD reduces the antioxidant enzyme activity and increases the lipid peroxidation in vivo (11).

Arterial compliance, also known as vascular compliance, is a prognostic indicator of arterial health (12). The impairment of the aortal function enhances vasoconstriction and weakens vasodilation (13). Usually, the response of the thoracic aorta vascular rings to NA reflects the arterial contractive function. Under the condition of the HD and aerobic exercise, the vascular elasticity and vasodilation are decreased by the excitation of the body's sympathetic nervous system $(14,15)$. In addition, the contractile response of the thoracic aorta to $\mathrm{KCl}$ is enhanced in rats fed with HD (16) and vascular compliance reduced (17). The present results showed that the contractile response of the aorta was increased in rats fed with HD. However, the continuous and intermittent exercises attenuated the increase of the contraction induced by NA. Furthermore, the effect induced by the intermittent exercise was stronger compared to the continuous exercise, which indicated that the intermittent exercise was more effective in protecting the aorta.

A previous study has shown that HD can increase the formation of endogenous free radicals and enhance the response to lipid peroxidation, which results in the impairment of the cell membrane (18). MDA is an oxidative product of free oxygen radicals with polyunsaturated fatty acids on the 
cell membrane, and it indicates the degree of lipid peroxidation (19). In addition, SOD is an important antioxidant enzyme that specifically removes superoxide anion radicals and protects cells, and its activity can indirectly reflect the body's ability to eliminate oxygen free radicals. Aerobic exercise can reverse the effect of the superoxide anion inside and outside the body (20). The effects of exercise on the activity of SOD are not consistent. Certain studies reported that exercise training improved the activity of SOD (21), and it is proposed that during exercise, the oxygen consumption and production of free radicals increase, which leads to the increase of intracellular antioxidant enzymes to produce acute adaptive changes (18). This process causes the body to produce antioxidants gradually. The effect of exercise on the activity of SOD is well-accepted as different due to the difference of the type intensity and durative time of exercise. In the present study, the MDA level was higher in the HD group, while the activity of SOD was decreased, indicating that HD enhanced lipid peroxidation induced by the endogenous oxygen free radicals. The present results showed that aerobic exercise significantly increased SOD activity and inhibited the myocardial MDA level in obese rats. In addition, the response of vascular rings in the thoracic aorta to NA is weakened in the HCE and HIE groups, suggesting that aerobic exercise can improve arterial function and vascular elasticity via its antioxidant effects. This is consistent with one study that showed the improvement of vascular elasticity by reducing oxidative stress (22).

Studies often use animal models of hyperlipidemia induced by HD for prevention research. In order to establish the hyperlipidemia model, SD rats are fed with HD recipes for 8 weeks and HD leads to lipid metabolism disorders involving the increase in serum TC, TG and LDL, as well as the decrease in HDL. These changes are major risk factors for cardiovascular disease, such as arterial injury (10,23,24). A previous study has shown that the high cholesterol-facilitated vasoconstriction response and the vascular reactivity to NA in various studies were different (25). The effects of exercise on cholesterol metabolism have been studied for years, although there were certain differences in the results due to the difference in exercise types, exercise intensity, research objective and the methods (26). A large number of epidemiological and experimental studies found that long-term regular exercise improved their poor lipid structures effectively, so that the risk of cardiovascular disease was significantly reduced (27). The present study also showed that the continuous and intermittent exercises affected the plasma lipid metabolism, decreasing the levels of LDL, TC and TG, while increasing the HDL level, which may be associated with increased lipoprotein lipase activity to release more fatty acids from lipoproteins (28).

A number of studies have been performed to investigate the effects of different intensities of exercise on eNOS in rat cardiomyocytes. Previous studies have shown that eNOS activity of the thoracic aorta was decreased in rats fed with HD and following long-term exercise training, the activity and mRNA expression of eNOS were upregulated (29-31). In addition, the elevation of eNOS gene expression and activity was observed in aortic endothelial cells, coronary blood vessels and cardiac capillaries in dogs that exercised persistently for 10 days. Wang et al (32) also found that eNOS activity of the thoracic aorta significantly increased in rats after
90 or 150 min of swimming training. Furthermore, the nitric oxide level in plasma was also significantly increased. The increase of eNOS activity in the 150 min training group was smaller than that in the 90 min training group $(33,34)$. In the present study, eNOS expression was significantly decreased in the HD group. Following the exercise, the expression of eNOS was significantly increased compared to that in the HD group and its increase was higher in the HIE group compared to the HCE group. These data indicate that aerobic training can enhance eNOS activity in myocardial cells, improve endothelial function and also delay the development of atherosclerotic plaques.

In conclusion, continuous and intermittent exercise can improve vasoreactivity of obese rats, which may be associated with improved exercise capacity, enhanced antioxidant enzyme activity and reduced free radicals and lipid peroxides, as well as increased gene expression of eNOS. In addition, the intermittent exercise had a better effect than the continuous exercise, but the detailed mechanisms of the regulation require further study.

\section{Acknowledgements}

The present study was supported by the Key Research Program of Ningxia Public Health Department (grant no. 2013114).

\section{References}

1. World Health Organization (WHO): Obesity: Prevention and management the global epidemic. Report of a WHO consultation. World Health Organization, Geneva, 1998.

2. Tock L, Prado WL, Caranti DA, Cristofalo DM, Lederman H, Fisberg M, Siqueira KO, Stella SG, Antunes HK, Cintra IP, et al: Nonalcoholic fatty liver disease decrease in obese adolescents after multidisciplinary therapy. Eur J Gastroenterol Hepatol 18: 1241-1245, 2006.

3. Rosa EC, Zanella MT, Ribeiro AB and Kohlmann O Jr: Visceral obesity, hypertension and cardio-renal risk: A review. Arq Bras Endocrinol Metabol 49: 196-204, 2005 (In Portuguese).

4. Westerterp KR: Perception, passive overfeeding and energy metabolism. Physiol Behav 89: 62-65, 2006.

5. Hambrecht R, Adams V, Erbs S, Linke A, Kränkel N, Shu Y, Baither Y, Gielen S, Thiele H, Gummert JF, et al: Regular physical activity improves endothelial function in patients with coronary artery disease by increasing phosphorylation of endothelial nitric oxide synthase. Circulation 107: 3152-3158, 2003.

6. Jia B, Wang X, Kang A, Wang X, Wen Z, Yao W and Xie L: The effects of long term aerobic exercise on the hemorheology in rats fed with high-fat diet. Clin Hemorheol Microcirc 51: 117-127, 2012.

7. Zhao Z-F, Min Y, Nan Z, et al: Influence of Lycium barbarum polysaccharides on thoracic aortic vascular reactivity and free radical metabolism at high temperature in exhaustive exercise rats. J Ningxia Med Coll 35: 481-484, 2013.

8. Jeppesen $\mathbf{J}$ and Kiens B: Regulation and limitations to fatty acid oxidation during exercise. J Physiol 590: 1059-1068, 2012.

9. Roberts CK, Vaziri ND, Liang KH and Barnard RJ: Reversibility of chronic experimental syndrome $\mathrm{X}$ by diet modification. Hypertension 37: 1323-1328, 2001.

10. Carroll JF, Thaden JJ, Wright AM and Strange T: Loss of diurnal rhythms of blood pressure and heart rate caused by high-fat feeding. Am J Hypertens 18: 1320-1326, 2005.

11. Mahapatra S, Padhiary K, Mishra TK, Nayak N and Satpathy M: Study on body mass index, lipid profile and lipid peroxidation status in coronary artery disease. J Indian Med Assoc 96: 39-40, 42, 1998

12. Laurent S, Cockcroft J, Van Bortel L, Boutouyrie P, Giannattasio C, Hayoz D, Pannier B, Vlachopoulos C, Wilkinson I and Struijker-Boudier H; European Network for Non-invasive Investigation of Large Arteries: Expert consensus document on arterial stiffness: Methodological issues and clinical applications. Eur Heart J 27: 2588-2605, 2006. 
13. Brandes RP, Behra A, Lebherz C, Böger RH, Bode-Böger SM, Phivthong-Ngam L and Mügge A: N(G)-nitro-L-arginine- and indomethacin-resistant endothelium-dependent relaxation in the rabbit renal artery: Effect of hypercholesterolemia. Atherosclerosis 135: 49-55, 1997.

14. Swierblewska E, Hering D, Kara T, Kunicka K, Kruszewski P, Bieniaszewski L, Boutouyrie P, Somers VK and Narkiewicz K: An independent relationship between muscle sympathetic nerve activity and pulse wave velocity in normal humans. J Hypertens 28: 979-984, 2010.

15. Ewert P, Berger F, Nagdyman N, Kretschmar O and Lange PE: Acute left heart failure after interventional occlusion of an atrial septal defect. Z Kardiol 90: 362-366, 2001 (In German).

16. Kim Y, Kim J, Kim M, Baek W and Kim I: Effect of heat shock on the vascular contractility in isolated rat aorta. J Pharmacol Toxicol Methods 42: 171-174, 1999.

17. Morimoto T, Miki K, Nose H, Itoh T and Yamada S: Changes in vascular compliance during hyperthermia. J Therm Biol 9: 149-151, 1984

18. Shan X, Zhou J, Ma T and Chai Q: Lycium barbarum polysaccharides reduce exercise-induced oxidative stress. Int J Mol Sci 12: 1081-1088, 2011.

19. Yan T, Oberley LW, Zhong W and St Clair DK: Manganese-containing superoxide dismutase overexpression causes phenotypic reversion in SV40-transformed human lung fibroblasts. Cancer Res 56: 2864-2871, 1996.

20. Cui S, Reichner JS, Mateo RB and Albina JE: Activated murine macrophages induce apoptosis in tumor cells through nitric oxide-dependent or -independent mechanisms. Cancer Res 54 2462-2467, 1994.

21. Jenkins RR, Martin D and Goldberg E: Lipid peroxodative in skeletal muscle during atrophy and acute exercise. Med Sci Sports Exerc 15: 93-94, 1983.

22. Mc Clean CM, Mc Laughlin J, Burke G, Murphy MH, Trinick T, Duly E and Davison GW: The effect of acute aerobic exercise on pulse wave velocity and oxidative stress following postprandial hypertriglyceridemia in healthy men. Eur J Appl Physiol 100: 225-234, 2007.

23. Hägg GM: Interpretation of EMG spectral alterations and alteration indexes at sustained contraction. J Appl Physiol (1985) 73: 1211-1217, 1992.
24. Zöllner N and Tatò F: Fatty acid composition of the diet: Impact on serum lipids and atherosclerosis. Clin Investig 70: 968-1009, 1992.

25. Verbeuren YJ, Jordaens FH, Zonnekeyn LL, et al: Effect of hypercholesterolemiar on vascular reactivity in the rabbit. I. Endothelium-dependent and endothelium-independent contractions and relaxations in isolated arteries of control and hypercholesterolemic rabbits. Circ Res 58: 552-564, 1986.

26. Abbott C, Meadows AB and Lier K: Low cholesterol and noncardiovascular mortality. Mil Med 165: 466-469, 2000.

27. Rybin VO and Steinberg SF: Protein kinase C isoform expression and regulation in the developing rat heart. Circ Res 74: 299-309, 1994.

28. Lira FS, Carnevali LC Jr, Zanchi NE, Santos RV, Lavoie JM and Seelaender M: Exercise intensity modulation of hepatic lipid metabolism. J Nutr Metab 2012: 809576, 2012.

29. Li R, Wang WQ, Zhang H, Yang X, Fan Q, Christopher TA, Lopez BL, Tao L, Goldstein BJ, Gao F, et al: Adiponectin improves endothelial function in hyperlipidemic rats by reducing oxidative/nitrative stress and differential regulation of eNOS/iNOS activity. Am J Physiol Endocrinol Metab 293: E1703-E1708, 2007.

30. Aoyama T, Takeshita K, Kikuchi R, Yamamoto K, Cheng XW, Liao JK and Murohara T: Gamma-Secretase inhibitor reduces diet-induced atherosclerosis in apolipoprotein E-deficient mice. Biochem Biophys Res Commun 383: 216-221, 2009.

31. Hecker M, Mülsch A, Bassenge E, Förstermann U and Busse R: Subcellular localization and characterization of nitric oxide synthase(s) in endothelial cells: Physiological implications. Biochem J 299: 247-252, 1994.

32. Wang J, Wu Y, Wang H, et al: The effect of sport fatigue on endothelium cell of rats and antioxidative protection of Tongxinluo super. Chin J Ethnomed Ethnopharmacy: 61-65, 2008.

33. Bora I, Seçkin B, Zarifoglu M, Turan F, Sadikoglu S and Ogul E: Risk of recurrence after first unprovoked tonic-clonic seizure in adults. J Neurol 242: 157-163, 1995.

34. Galski T, Ehle HT and Williams JB: Off-road driving evaluations for persons with cerebral injury: A factor analytic study of predriver and simulator testing. Am J Occup Ther 51: 352-359, 1997. 